\title{
Microscopic pulmonary embolisation of an indwelling central venous catheter with granulomatous inflammatory response
}

\author{
A. Baydur*, M.N. Koss”, O.P. Sharma*, G.E. Dalgleish", D.V. Nguyen, \\ F.G. Mullick ${ }^{\S}$, L.A. Murakata ${ }^{\S}$ and J.A. Centeno ${ }^{\S}$
}

ABSTRACT: Indwelling catheters can disintegrate into tiny fragments and embolise. Once the fragments are detected radiographically, they can be removed using vascular intervention techniques. Rarely, indwelling catheters dwindle into inextricable pieces that embolise into minute pulmonary vessels and lymphatics, causing granulomatous changes microscopically.

The present study reports a 54-yr-old female who had received several indwelling central lines during several abdominal surgeries over a 5-yr period. The patient developed a noncaseating granulomatous skin lesion followed by exertional dyspnoea a few months later. Chest radiographs and computed tomography showed diffuse interstitial infiltrates. Open lung biopsy showed two types of granulomas: 1) peri-lymphangitic and peri-bronchiolar non-necrotising granulomas consistent with sarcoidosis; and 2) distinct foreign body granulomas.

In some of the foreign body granulomas, confocal Raman spectroscopy identified the presence of bisphenol-A-polycarbonate, a polymer commonly used in biomedical devices. The patient improved following treatment with prednisone followed by methotrexate.

The present case illustrates an interesting combination of two causes of granulomatous disease, the importance of examining all biopsy specimens from sarcoidosis patients for foreign particles and the rare occurrence of microscopic embolisation of catheter fragments to the lung with foreign-body giant cell reaction to them.

KEYWORDS: Bisphenol-A-polycarbonate, catheter embolisation, catheter fragmentation, foreignbody granulomas

$\mathbf{T}$ hromboembolism associated with the use of indwelling catheters is well-known; however, spontaneous catheter fragmentation causing embolisation is infrequent [1-5]. The present study reports a case in which catheter particles embolised into pulmonary vessels and produced a granulomatous inflammation. This foreign body reaction occurred in the setting of multisystem sarcoidosis.

\section{CASE REPORT}

A 54-yr-old female with asthma and hypothyroidism was evaluated for dyspnoea of several months' duration. From 1969 through to 1998, she had undergone a total abdominal hysterectomy, subsequent bowel resection for obstruction (due to adhesions, ureteral obstruction and cholecystectomy) and multiple hospitalisations for intestinal obstruction. From 1994 through to 1998, she underwent insertion and replacement of at least five different indwelling central venous catheters. Late in 2002, the patient began to experience arthralgias and developed a skin lesion on her leg, which was biopsied and revealed noncaseating granulomas. The serum angiotensinconverting enzyme level was $60 \mathrm{U} \cdot \mathrm{L}^{-1}$ (normal levels 8-67 U. $\mathrm{L}^{-1}$ ). Pulmonary function testing carried out in August 2003 showed a forced vital capacity (FVC) 97\% predicted, forced expiratory volume in one second (FEV1)/FVC ratio of $79 \%$, total lung capacity of $102 \%$ pred and a corrected single-breath carbon monoxide diffusion capacity of $73 \%$ pred. There was no reversal in flow rates following inhalation of bronchodilator. Chest radiographs exhibited bilateral reticulonodular markings, with gallium scanning of the lungs showing diffuse uptake. Computed tomography
AFFILIATIONS

Depts of *Internal Medicine, and \#Dept of Pathology, Division of Pulmonary and Critical Care Medicine, Keck School of Medicine, University of Southern California, Los Angeles, and

"Dept of Pathology, and

${ }^{+}$Division of Pulmonary and Critical Care Medicine, Simi Valley Hospital, Simi Valley, CA, and

${ }^{\S}$ Dept of Environmental and Infectious Disease Science, Division of Biophysical Toxicology, Armed Forces Institute of Pathology, Washington, DC, USA.

\section{CORRESPONDENCE}

A. Baydur

Professor of Medicine Division of Pulmonary and Critical

Care Medicine

Keck School of Medicine 2025 Zonal Avenue, GNH 11-900 Los Angeles

CA

USA

Fax: 13232262738

E-mail: baydur@usc.edu

Received:

November 232004

Accepted after revision:

March 082005

European Respiratory Journal Print ISSN 0903-1936 Online ISSN 1399-3003 
of the lungs with contrast showed bilateral linear densities consistent with parenchymal fibrosis. There was no alveolitis, or mediastinal or hilar adenopathy. Transbronchoscopic lung biopsy was nondiagnostic. In October 2003, a wedge biopsy of the left lower lobe showed two types of granulomas: 1) noncaseating granulomas with no foreign bodies; and 2) foreign-body granulomas with birefringent particles (figs 1 and 2). Gomori methenamine silver stain for fungi and the Ziehl-Nielsen stain for acid fast bacilli were negative for organisms. Other microscopic findings included: muscular hypertrophy of bronchioles, consistent with the patient's history of asthma; peribronchiolar glandular metaplasia, indicative of small airways disease; and mild emphysema.

To determine the origin of the foreign material, unstained microscopic slides containing several foreign-body granulomas were sent to the Dept of Environmental and Infectious Disease Science at the Armed Forces Institute of Pathology (Washington, DC, USA). There, the birefringent material seen in several of the foreign-body granulomas was examined by $\mathrm{J}$. Centeno and L. Murakata, using confocal laser Raman microscopy (fig. 2), and was identified as bisphenol-Apolycarbonate, a polymer widely used in many industrial and biomedical applications, including catheters.

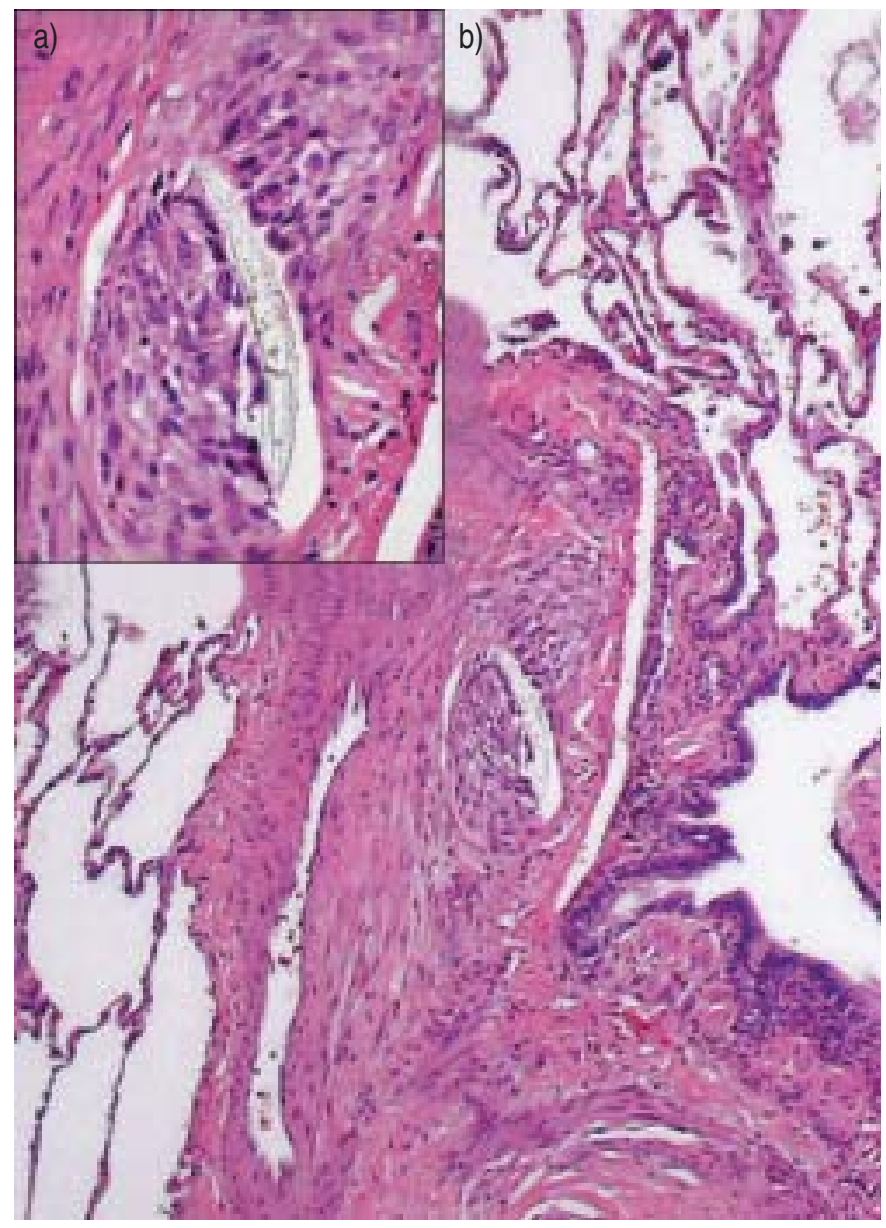

FIGURE 1. a, b) Left lower-lobe wedge biopsy of the lung. Non-necrotising granuloma containing birefringent material within the wall of a hypertrophic pulmonary arteriole (enlarged centre of figure shown in a).
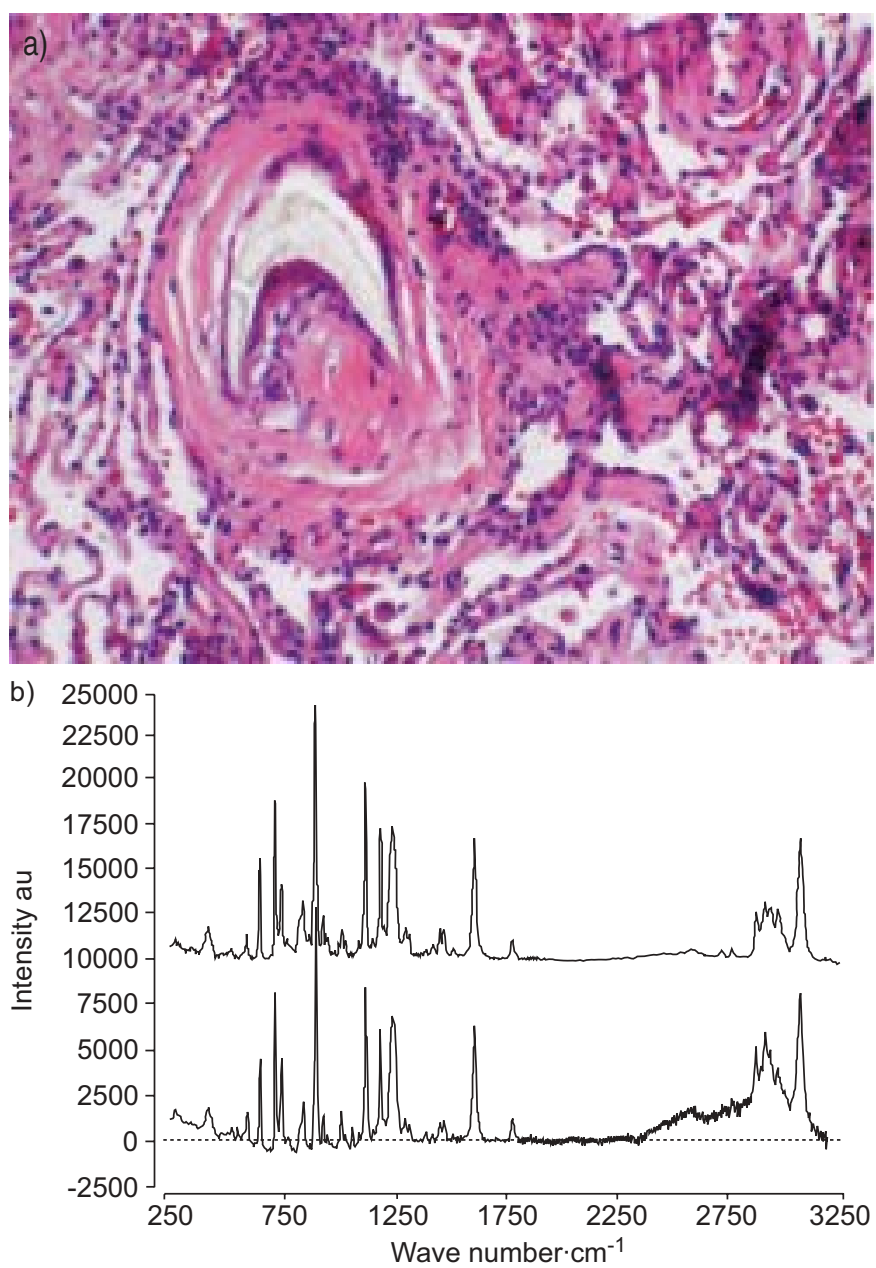

FIGURE 2. a) Confocal laser Raman spectroscopic identification of the birefringent material as bisphenol-A-polycarbonate in tissue, and b) the reference spectral trace for this agent in the tissue. The top reading correlates to poly (bisphenol-A) carbonate reference and the lower reading to poly (bisphenol-A) carbonate in tissue.

The patient was treated with prednisone, leading to improvement in dyspnoea. When the patient developed corticosteroidrelated symptoms, the prednisone was discontinued and weekly methotrexate was started. To date the patient remains stable, and the serum angiotensin-converting enzyme level has decreased to $27 \mathrm{U} \cdot \mathrm{L}^{-1}$.

\section{DISCUSSION}

Indwelling catheters are commonly used for long-term parenteral nutrition in debilitated patients and for chemotherapy in patients with malignancy. Rarely, indwelling central venous catheters spontaneously disintegrate and embolise. The incidence of such an occurrence is $0.1 \%$ [1]. A large shower of particles can cause physiological changes similar to that of foreign-body emboli in intravenous drug users. The events leading to the break-up of the catheter include kinking and shearing related to repeated insertions and removals [1-5].

To the current authors' knowledge, the present study is the only case in which the catheter fragments were discovered unexpectedly on histological examination during investigation 
of an interstitial process. Two distinct types of granulomas were found. The authors believe that the two granulomatous processes were unrelated. The foreign-body granulomas that revealed bisphenol-A-polycarbonate, a common biomedical polymer determined by confocal Raman spectroscopy, were caused during 5 yrs of repeated hospitalisations while the patient remained clinically asymptomatic.

After remaining stable and asymptomatic for $8 \mathrm{yrs}$, the patient developed cutaneous lesions, the first evidence of multisystem sarcoidosis. The diagnosis was established on the basis of finding typical granulomas in skin and lung tissue, a mildly abnormal serum angiotensin-converting enzyme level and a positive gallium uptake i.e. increased inflammatory activity [6]. The patient responded to prednisone and methotrexate and has since remained stable.

The present case underlines the importance of examining all biopsy specimens from sarcoidosis patients for foreign-body particles and chemical analysis. Furthermore, in order to keep the incidence of indwelling catheter fragmentation and embolisation low, attention should be paid during insertion and removal of indwelling catheters.

\section{REFERENCES}

1 Roye DG, Breazeale EE, Byrnes JP, et al. Management of catheter emboli. Southern Med J 1996; 89: 714-718.

2 Rubenstein RB, Alberty RE, Michels LG, et al. Hickman catheter separation. J Parenter Enteral Nutr 1985; 9: 754757.

3 Propp DA, Cline D, Hennenfent BR. Catheter emboli. J Emerg Med 1988; 6: 17-21.

4 Schmierer JA, Isner JM, Konstam MA, et al. Catheter fragmentation: a potential source of catheter embolus. Cathet Cardiovasc Diagn 1983; 9: 595-599.

5 Prager D, Hertzberg RW. Spontaneous intravenous catheter fracture and embolization from an implanted venous access port and analysis by scanning electron microscopy. Cancer 1987; 60: 270-273.

6 Statement of sarcoidosis: Joint statement of the American Thoracic Society (ATS), the European Respiratory Society (ERS) and the World Association of Sarcoidosis and Other Granulomatous Disorders (WASOG) adopted by the ATS Board of Directors and by the ERS Executive Committee, February 1999. Am J Respir Crit Care Med 1999; 160: 736-755. 